\title{
Las Controversias Históricas en Psicología: ¿Anomalías Irracionales Egotistas o Instancias Estructurales de Debate Racional?
}

\section{Historical Controversies in Psychology: Irrational Egotistic Anomalies or Structural Stages of Rational Debate?}

\author{
Catriel Fierro ${ }^{2}$ \\ Facultad de Psicología, Universidad Nacional de Mar del Plata, Buenos Aires, Argentina
}

(Rec: abril 2015 - Acept: mayo 2015)

\begin{abstract}
Resumen
El presente trabajo expone un relevamiento empírico de carácter histórico acerca de la inserción, definición y valorización de controversias científicas en psicología en función de ciertas tendencias historiográficas en psicología. Con el objetivo general de revisar la importancia de las instancias controversiales para el desarrollo científico de la psicología, y el objetivo específico de posibilitar acercamientos entre la historiografía de la psicología y los estudios sociales de la ciencia, se describe primero la definición y explicación de las controversias históricas realizadas por la historiografía clásica de la psicología, enfatizándose el carácter anómalo de tales controversias para dicha historiografía dados sus implícitos teórico-metodológicos. Se detalla luego la redefinición realizada en torno a controversias históricas por la 'nueva historia' de la psicología. Se remarca que tal redefinición se debió en parte a la incorporación por los historiadores de ciertos principios y recursos explicativos sociológicos de la historia social de la ciencia y de los estudios sociales de la ciencia. Se concluye sobre el carácter productivo de tal redefinición y de las prospectivas de profundizar en análisis socio-históricos de controversias psicológicas.

Palabras clave: historiografía de la psicología, historia social de la psicología, estudios sociales de la ciencia, controversias científicas, programa empírico del relativismo.
\end{abstract}

\begin{abstract}
This paper presents an empirical (historical) survey about the insertion, definition and valuation of scientific controversies in psychology on the basis of certain historiographical trends in History of Psychology. With the overall objective of reviewing the importance of controversial instances for the scientific development of psychology, and the specific objective of enabling rapprochement between the historiography of psychology and social studies of science, the definition and explanation given about historical controversies by the classic historiography of psychology are first described, emphasizing the anomalous character of such controversies to said historiography given the implicit theoretical and methodological principles of such tradition. The redefinition of historical controversies by the 'new history' of psychology developed since 1970 is then detailed. It is noted that such redefinition took place partly due to the historians' incorporation of certain sociological and socio-historical principles and explanatory resources from social history of science and social studies of science. The positive character of such a redefinition and of the foresight of deeper socio-historical analysis of controversies in psychology is then concluded.
\end{abstract}

Keywords: historiography of psychology, social history of science, social studies of science, scientific controversies, empirical programme of relativism

\footnotetext{
Este artículo está asociado al proyecto de investigación "Formación de psicólogos. Enseñanza de la historia de la psicología y estudio de áreas de vacancia", código 15/H224, de la Universidad Nacional de Mar del Plata, Argentina.

2 Correspondencia a: Catriel Fierro. Universidad Nacional de Mar del Plata, Facultad de Psicología. Dean Funes 3250 - Cuerpo II - Nivel III, sala "Alberto Vilanova". Mar del Plata. Buenos Aires. Argentina. E-mail: catriel.fierro@ gmail.com.
} 


\section{La Controversia Científica en Psicología: entre la Historia de la Ciencia y los Estudios Sociales de la Ciencia y la Tecnología}

Desde hace cinco décadas, el estudio de la gesta, producción, difusión y socialización del conocimiento científico registra un crecimiento constante. El campo académico de tales estudios, la 'sociología de la ciencia' contemporánea, es identificado usualmente con el campo de los 'estudios sociales de la ciencia y la tecnología' (Bucchi, 2004), especialmente cuando la primera no se reduce a la perspectiva mertoniana. Las propuestas programáticas al respecto son extremadamente disímiles y variadas, lo que se evidencia al constatar que la propia empresa científica puede identificarse con una institución, con un producto cognoscitivo o como una actividad humana socialmente regulada (Gallegos, 2014; Jaraba-Barrios \& Mora-Gámez, 2010). La presente investigación pretende recuperar un marco analítico específico de dichos estudios -los análisis sociológicos de controversias científicas del 'Programa Empírico del Relativismo'- con el fin de remarcar afinidades, entrecruzamientos y potenciales enriquecimientos mutuos entre la sociología de la ciencia y la historia de la psicología, definida esta como una especialidad investigativa empírica al interior de la psicología y lindante con la historia de la ciencia.

Definidas como aquellos debates en torno a enunciados contrapuestos que académicos y científicos emiten en calidad de descripciones o juicios acerca de objetos o problemáticas científicas, las controversias científicas evidencian a criterio de los sociólogos la naturaleza social de parte de la dinámica científica. Según Collins y Pinch (1993), tales instancias demuestran la naturaleza en principio abierta de la evidencia y del conocimiento científico, toda vez que estas estarían abiertas a múltiples interpretaciones. Dado el carácter interpretable de los resultados de las investigaciones y de sus replicaciones, la forma en que las controversias científicas se resuelven ilustraría tanto la determinación del conocimiento por parte de las actividades sociales y retóricas de los científicos, como la naturaleza noepistémica de algunos de los factores determinantes de dicho conocimiento (Collins, 1981, 1983). Factores sociales, como "la reputación del experimentador y su institución, su nacionalidad, su estatus en su comunidad de investigación particular [o] las opiniones informales de sus colegas" (Bucchi, 2004, p. 68), o factores económicos, políticos y culturales, serían factibles de incidir sobre la modalidad, el desarrollo y el cierre de las controversias. De aquí que para cada controversia se analice la flexibilidad interpretativa de los resultados de experiencias científicas, los mecanismos que ponen fin a la recursividad a que lleva la flexibilidad interpretativa, y la conexión entre estos mecanismos de cierre y la estructura social más general (de la ciencia o del subgrupo específico de científicos envueltos en la controversia).

Gran parte de las controversias científicas analizadas por los estudios sociales de la ciencia pertenecen al pasado científico (Fernández, 2009). La consideración de polémicas y controversias científicas como objetos legítimos de indagación por parte de los estudios sociales de la ciencia fue precedida por la nueva historiografía de la ciencia de los '60 (Buchdahl, 1965), que en términos de revoluciones, discontinuidades y anomalías enfatizó la naturaleza controversial y problemática tanto de la producción como de la difusión y aceptación del conocimiento científico (Brush, 1974; Kuhn, 1962/1970). Dicha 'nueva historia' se desarrolló posteriormente, entre otras cosas, en historia social o sociológica de la ciencia y la tecnología (Golinski, 1990; Schaffer, 1985; Shapin, 1982).

Si bien el vínculo entre los estudios sociales de la ciencia y la historia e historiografía de la ciencia es claro y definido (Ziman, 1985), el vínculo entre los estudios sociales de la ciencia y la historia de la psicología como disciplina investigativa es débil y, a menudo, predominantemente implícito. Sólo hacia 1980 la historiografía de la psicología comenzó a incorporar sistemáticamente recursos heurísticos, explicativos y de análisis propios de la filosofía y la sociología de la ciencia post-kuhniana (Capshew, 2014; Fierro, 2015b). Sin embargo, la historia social de la psicología ha sido considerada en escasa medida por los psicólogoshistoriadores (Ash, 1980; Danziger, 1994; Richards, 1987). Contribuyendo su parte al aislamiento entre ambos campos, los sociólogos de la ciencia y los más contemporáneos social studies han priorizado análisis de 'ciencias duras' (como la física, la biología y la medicina), lo que ha relegado a la historia de la psicología como objeto de análisis (Ash, 1992; Diéguez, 2004).

Considerada la naturaleza controversial -polémica o escolástica- de gran parte del desarrollo histórico de la disciplina (Buss, 1978), se propone analizar y revisar, a partir de un relevamiento empírico de fuentes documentales primarias y secundarias, las propuestas historiográficas desde las cuales se han analizado las controversias históricas en psicología. Tales propuestas refieren, por un lado, a la historiografía 'clásica' (de primera mitad de siglo XX) y por otro, a la corriente de renovación de dicha historiografía clásica, denominada 
'revisionista' o 'crítica' (Danziger, 1984; Woodward, 1980), estimulada por la profesionalización de los historiadores (Capshew, 2014) y por la recepción de teorías y metodologías novedosas de la filosofía y la sociología de la ciencia (Polanco \& Fierro, 2015; Talak, 1997). Este relevamiento y análisis histórico y meta-científico persigue el objetivo general de revisar la importancia de las instancias controversiales para el desarrollo científico de la psicología y el objetivo específico de posibilitar acercamientos entre los campos disciplinares de la historiografía de la disciplina y de los estudios sociales de la ciencia. La indagación 'meta-científica' que se propone implica una focalización en las nociones teóricas y metodológicas a que echaron mano los historiadores para abordar ciertas controversias históricas concretas ${ }^{3}$. Dado que el objeto de la presente investigación es el tratamiento de las controversias a partir de las corrientes historiográficas aludidas, las fuentes primarias que aquí se abordan constituyen principalmente las obras de los historiadores (Klappenbach, 2000). Dado que hasta mediados de siglo XX en Norteamérica las obras y cursos históricos se confundían con obras y cursos de sistematología psicológica o de psicología contemporánea (Fierro, en prensa; Klappenbach, 2000), adoptaremos aquí una definición pragmática de aquella historiografía (Vilanova, 1995, 1997a), y de acuerdo a múltiples relevamientos sistemáticos previos ubicaremos a Edwin Boring como representante y ejemplar cabal de dicha historiografía clásica, seguido por autores como Brett, Murphy y Woodworth (Ash, 1983; Buss, 1975; Danziger, 1990; Louw, 2003), garantizando así representatividad en las conclusiones extraídas del análisis.

Finalmente, el análisis propuesto implica una cuestión propedéutica: el supuesto de que la reflexión acerca de la historia de la ciencia colabora con el

\footnotetext{
De acuerdo a la aplicación sobre estas cuestiones del modelo meta-científico de práctica histórica de construcción teórica (van Strien, 1993), los historiadores de la psicología han constituido durante el siglo XX un sub-grupo particular de la comunidad disciplinar cuya práctica (la producción de conocimiento histórico) constituye la respuesta operativa a problemas conceptuales (abstractos) y materiales (concretos) específicos originados en la práctica docente (en la socialización de los estudiantes) y en la necesidad de justificar la investigación contemporánea (finalismo). Hacia 1930 los historiadores anglosajones, enmarcados en un contexto institucional experimentalista y empirista, legitimaron su propio trabajo a partir de una reconstrucción parcial y orientada de la historia disciplinar. En dicho marco, el abordaje de las controversias históricas se volvió una cuestión problemática. Las herramientas conceptuales adoptadas por los historiadores para la resolución de tal problemática provinieron del contexto intelectual y cultural de la psicología, no del de la historia e historiografía de la ciencia (Wettersten, 1975).
}

mejoramiento de la formación académica y profesional del psicólogo. Tal historia, en tanto empresa de indagación empírica y crítica del pasado, y componente esencial del currículo del psicólogo, es susceptible de estimular tal mejoramiento académico al priorizar narrativas históricas complejas (no necesariamente lineales) y multideterminadas (esto es, no guiadas intrínsecamente ni reducidas a concatenaciones de ideas y conceptos psicológicos), especialmente en latitudes académicas que, como la argentina, han rendido culto a la certeza cognoscitiva más que al carácter conjetural del conocimiento (Vilanova, 1997b).

\section{Las Controversias Históricas como Anomalías en la Historiografía Clásica de la Psicología}

Un análisis de la historia y la sistemática de la psicología de primera mitad de siglo XX demuestra que las controversias psicológicas no constituyeron para la historiografía clásica un objeto de estudio delimitado o de particular relevancia: las polémicas científicas aparecen allí minimizadas y recortadas, y sus implicancias no son desarrolladas. Esto responde a los implícitos de tal historiografía. En primera instancia la narrativa general expuesta en los clásicos manuales ha sido característicamente justificacionista, persiguiendo la finalidad de fundamentar las elecciones teóricas y metodológicas de los psicólogos contemporáneos más que la de retratar de forma sistemática y objetiva el desarrollo y la dinámica (policéntrica, problemática y en ocasiones recesiva) de la psicología (Blight, 1981; Weimer, 1974, 1979). Esto habría llevado a los historiadores clásicos a reconstruir de forma claramente selectiva -y distorsiva- el pasado disciplinar.

Adicionalmente, se ha sostenido que la historia clásica ha sido presentista, o en términos semejantes, whiggista. Sesgo propio de parte de la historiografía de la ciencia (Brush, 1974; Fierro, 2015a), el whiggismo, al relevar el pasado a partir de una valoración contemporánea en términos dicotómicos de 'acertados' y 'desacertados', necesariamente condena al error y al destierro de la historia y de la teoría a aquellas propuestas científicas que no son aceptadas consensuadamente en la actualidad. Las historias whiggish ignoran que variables tales como la difusión o socialización del conocimiento científico, o el propio clima y contexto intelectual de cada época socio-histórica puede de hecho modular la 'aceptación' o el 'rechazo' de una propuesta psicológica programática (Vilanova, 1990), 
haciendo que recursivamente tales propuestas psicológicas no aceptadas en el presente, y precisamente por el hecho de no serlo, se consideren sobrantes de las narrativas históricas.

Finalmente la historia de Boring y sus coetáneos ha sido definida como celebratoria o laudatoria (Furumoto, 1989; Harris, 1980; Woodward, 1980): esto es, como una historia que, especialmente ante los centenarios, prioriza la celebración en desmedro de la apreciación pormenorizada, crítica y contextualizada de la disciplina. Lo discutible a ojos de los historiadores más contemporáneos no es la celebración misma de los centenarios, sino la limitación autoimpuesta a reiterar y reproducir en tales centenarios lo que las historias clásicas han promulgado en términos de doctrinas y ortodoxias, sin escrutinio histórico crítico y reflexivo (Danziger, 1994).

Debe notarse que estos sesgos característicos de la historiografía clásica de la psicología son también sesgos característicos de la clásica historiografía de la ciencia (Brush, 1974): práctica que ha sido más ciencia (exposición teórica, metodológica o meramente descriptiva) que historia (narrativa reconstructiva y significativa de teorías, corrientes y acontecimientos institucionales y profesionales en un marco cultural y social).

A la luz de los sesgos descriptos, considerada la pertenencia profesional de los primeros historiadores de la psicología, y reconocida la función legitimante y socializadora las clásicas obras de historia, se sigue de suyo que las controversias científicas constituyeron 'anomalías' para la historiografía clásica: esto es, instancias que implican el reconocimiento de que "la naturaleza ha violado de alguna forma las expectativas derivadas del paradigma que gobiernan la ciencia" (Kuhn, 1962/1970, pp. 52-53). En tal sentido restringido, si identificamos el conjunto de premisas teóricas y metodológicas de la historiografía clásica arriba descritas con los componentes teóricos y metodológicos de un paradigma ${ }^{4}$, entonces la existencia de instancias controversiales y polémicas en el desarrollo histórico de la ciencia psicológica constituye claramente una anomalía.

En primer lugar, la existencia de representaciones $\mathrm{y}$ de pretensiones de conocimiento diversas en torno a los mismos objetos o sucesos psicológicos mina los

\footnotetext{
4 La identificación se realiza con fines heurísticos y expositivos. Aquí 'paradigma' refiere a los lineamientos teóricos y metodológicos ya descritos de la historiografía clásica, aceptados implícitamente por el conjunto de historiadores-sistematólogos de primeras décadas de siglo.
}

implícitos naturalistas y positivistas de la historiografía clásica (Danziger, 1990), puesto que problematiza la existencia de una realidad completamente independiente del observador (sea dicho observador un experimentalista decimonónico o un historiador contemporáneo). En segundo lugar, la existencia de controversias históricas mina la linealidad progresiva implícita en la historiografía clásica: dado que existen reportes radicalmente distintos sobre hallazgos aparentemente semejantes, y dado que tales hallazgos llevan a movimientos y escuelas radicalmente disímiles, la historia -ya no inexorablemente progresiva- es susceptible de ser objeto de regresiones y de modulaciones por factores extracientíficos. En tercer lugar, las controversias constituyen contraargumentos a la metafísica histórica del Zeitgeist: el determinismo idealista y supra-humano propuesto por esta noción hegeliana es refutado por la aparición concreta y situada de agentes sociales que, en contra del 'espíritu de los tiempos', interpretan cierta evidencia científica de forma disímil a como lo hacen otros científicos. Adicionalmente, cuando al Zeitgeist se le supone cierto sentido de dirección y de realización supremo e impersonal, tal como en ocasiones realiza Boring (Boring, 1955; Friedman, 1967; Ross, 1969), se imputa de hecho a la historia con un teleologismo cuyo rechazo es el precepto metodológico que demarca la historia como empresa filosófica y literaria de la historiografía profesional y científica (Capshew, 2014; Woodward, 1980). De aquí que Ross (1969) sostenga que "sin examinar el panorama histórico total, no hay razón para asumir a priori que ciertas tendencias intelectuales son el principal factor causal en algún evento o serie de eventos en la historia de la psicología" (pp. 262).

\section{Domesticando las Anomalías Históricas}

Las anomalías que para la historiografía clásica constituyen las controversias históricas en psicología han sido objeto de intentos resolutivos variados.

En primer lugar, las controversias a menudo han sido omitidas de las reconstrucciones históricas de la psicología, o -lo que a efectos prácticos es idénticohan sido presentadas de forma diluida. A pesar de que los historiadores y sistematólogos no escatimaron en tinta al momento de definir el estado fragmentario y escolástico de la disciplina (Klappenbach, 2013), ninguno de ellos analizó o tematizó de forma detallada en sus obras históricas generales los choques y las discusiones entre las escuelas teóricas, derivando y 
analizando explícitamente las implicancias epistemológicas y organizacionales más generales de dichos choques. Admitido el escolasticismo como el estado del campo psicológico durante las primeras décadas del siglo XX, pero procurando minimizar el efecto disruptivo del mismo:

¿De qué forma puede retratarse a la historia de la psicología como un desarrollo constante que culmina con la teoría contemporánea? [...] [A través de] evitar las discusiones de las controversias, errores y problemas; evitación que lleva a una imagen engañosa de la historia de la psicología científica. (Wettersten, 1975, pp. 157-158)

Las historias de la psicología de los años '30 de autores como Woodworth, Murphy, Boring, Kantor, Pillsbury y Mora oscilaban entre circunscribir la historia a una sola escuela -que coincidía a menudo con la escuela a que pertenecía el historiador, lo que sesgaba la reconstrucción histórica (Gruba-McCallister, 1978) - o en considerar todas las escuelas psicológicas sin reparar de forma detallada en las controversias explícitas e implícitas que se derivaban de la existencia de puntos de vista radicalmente los mismos fenómenos y objetos psicológicos. Esta última alternativa constituyó el camino más recorrido por los historiadores. Así, Murphy (1929/1964) reconstruía los antecedentes 'prehistóricos' de la psicología para luego desembocar en una exposición breve de varios sistemas psicológicos contemporáneos, sin atender explícitamente a las implicancias científicas de la fragmentación y pluralidad de la disciplina. Heidbreder (1933/1960), si bien con mayor sensibilidad epistemológica, luego de una introducción acerca de la psicología pre-científica exponía de forma general sistemas psicológicos desde un enfoque marcadamente personalista e individualista (esto es, centrado en los 'líderes de escuela'). Finamente, Woodworth (1938/1961) se decantaba por una exposición generalista de las escuelas psicológicas de 1930, sin vincularlas significativamente ni tematizar la cuestión de la diversidad y los frecuentes debates inter-escolásticos.

El estado fragmentario y superficial de tales reconstrucciones históricas fue notada por historiadores de la ciencia profesionales, de los cuales uno sostuvo que los historiadores de la generación de Boring tendieron a "escribir historia expositiva, dejando afuera los cabos sueltos y las preguntas provocativas que estimulan la investigación [...] La historia, como la ciencia, es controversia, no contar cuentos [story-telling]" (Young, 1966, pp. 16-17)

Así, respecto del conductismo watsoniano es constatable la deserción de las preguntas auténticamente problemáticas que surgen del choque teórico y metodológico de tal escuela con el resto de la psicología, optándose en cambio por descripciones superficiales y cronológicas:

¿Cuál es la teoría del conductismo? ¿Cuál es la justificación de la teoría o qué problemas resuelve? ¿Cuál es el resultado de su aplicación? ¿A qué conflictos dio lugar (por ejemplo, con Thorndike)? [...] Estas preguntas son ignoradas. Algunos historiadores admiten que las escuelas existieron y que Watson lideró una escuela. Pero la discusión acerca del trabajo de Watson se reduce a su aprobación del conductismo, y a veces a la alabanza de los logros del conductismo, como también a una discusión de influencias políticas o sociológicas, o a al éxito de la perspectiva conductista. (Wettersten, 1975, p. 166)

Efectivamente, Murphy (1929/1964) limitaba su exposición del conductismo a la reflexología y a una exposición teórica del conductismo watsoniano y sus innovaciones, sólo aludiendo a los 'debates' que provocaron las ideas radicales de dicha escuela. Y Heidbreder (1933/1960) exponía el conductismo a partir de identificar dicha escuela con la obra de John Watson, pero refiriendo en escasa medida a las discusiones con conexionistas, funcionalistas e introspeccionistas en general. No existiría en estas historias una tematización concreta y explícita de los choques y enfrentamientos entre escuelas psicológicas, desde una perspectiva teórica (internalista) o epistemológica (externalista): más bien, existiría una exposición noproblemática de puntos de vista.

El único historiador clásico que parece haberse interesado en las controversias como objeto de análisis per se fue el propio Boring, quien en su obra tardía mostró interés por incorporar ciertas nociones de sociología y filosofía de la ciencia a la historia de la psicología

\footnotetext{
5 Es encomiable la actitud no-partidaria de los autores referidos al momento de optar por presentar en pie de igualdad a todas las escuelas y sistemas psicológicos, dejando de lado caras alianzas escolásticas (lo cual no puede decirse respecto de Boring, fuertemente experimentalista). Lo cuestionable de tales reconstrucciones, a la luz de la actualidad, es precisamente y como remarca Young, el carácter expositivo (no problematizador) de tales historias. En este sentido consúltese Fierro y Visca (en prensa) y Klappenbach (2013).
} 
(Gruba-McCallister, 1978; Young, 1966). Sin embargo, tal abordaje fue realizado principalmente en artículos aislados, sin incorporación a su obra específicamente histórica, la cual exponía escuelas pero no debates o, más propiamente, controversias (Klappenbach, 2013). En este respecto es notable el contraste entre la inespecificidad de su manual histórico y valoración que realizaba (en su discurso de presidencia ante la APA) en torno al escolasticismo en el año de publicación de aquel: "Los nuevos movimientos en psicología [...] son mayoritariamente negativos. Lo que pretende hacerse pasar por progreso, aquello que es aceptado como tal en el presente, es sin embargo y de forma patente la anulación [undoing] del progreso del pasado" (Boring, 1929/1963b, p. 78).

En este sentido, cuando las controversias han sido tematizadas por los historiadores, los análisis realizados han sufrido de dos principales limitaciones: han sido mayoritariamente descritas (puesto que no explicadas), y tales descripciones casi exclusivamente se ha realizado en términos psicológicos.

Al definir las causas de las controversias científicas, Boring apunta exclusivamente a factores individuales y psicológicos. En sus propios términos, “¿Qué crea la controversia científica? La interferencia del ego, el sesgo del ego. Nunca se encuentra a dos científicos en amarga controversia porque cada uno cree que el otro está en lo correcto y él está equivocado" (Boring, 1954, p. 639). Aunque el Zeitgeist también incidiría en las controversias, puesto que "una fuerza prejuiciosa más sutil y en ocasiones más siniestra puede hallarse en la atmósfera intelectual que recubre el pensamiento del científico" (Boring, 1954, pp. 639-640), lo determinante en la causación y dinámica de la controversia es el involucramiento emocional e irracional del científico (Boring, 1929/1963a; Friedman, 1967). Factores individuales, emocionales y psicodinámicos estarían en el núcleo de la actividad científica:

Del egoísmo se extrae el impulso y el entusiasmo que lleva a los hombres a encarar investigaciones, a mantenerse en ellas, a publicar los resultados, a continuar promoviendo el conocimiento y a usar dichos resultados. También del egoísmo se deriva el apoyo emocional [...] para cada científico que aún se aferra a una teoría hasta luego de que el peso de la evidencia ya no lo justifica. (Boring, 1954, p. 640)

Tal egoísmo (o egocentrismo) es uno de los principios rectores que, junto con el fervor religioso, las creencias y la necesidad de aprobación, configuran según Boring los 'pequeños Zeitgeist' que constituyen las escuelas psicológicas.

El egocentrismo lleva a la verdad. También explica la hipérbole y la exageración del investigador entusiasta, y por tanto puede llevar a la falsedad. Cuando dos egoísmos incompatibles se encuentran, se explica el tiempo perdido de la contienda científica, debido al destronamiento de la razón por parte de la racionalización. (Boring, 1955, p. 105)

Para Boring, serían factores exclusivamente psicológicos los que conducirían a las controversias en psicología: egoísmo, interés por la prioridad, necesidad de prestigio, creencia y fe en la temática de la escuela de pertenencia, impulsos inconcientes, entre otros (Boring, 1929/1963b). Contra la incidencia grupal y social, Boring prioriza explicaciones psicologistas: las mentes que se encuentran en una controversia están 'cerradas' por el orgullo y el prejuicio. Este es el punto más débil de la postura boringniana: el sostener que los debates que han trabado los psicólogos a lo largo de la historia son productos de sesgos interpretativos indeseables y factibles de ser evitados, más que resultados de la naturaleza estructural de ciertos sesgos de la cognición humana y de la naturaleza conflictiva de la dinámica grupal y social de la ciencia psicológica. Esto desemboca en narraciones superficiales y psicologistas de las controversias: los análisis acerca de las controversias en torno a autores históricamente significativos como Mesmer, Wundt y Stumpf, y Baldwin y Titchener, se centran precisamente en las 'tendencias egotisticas', auto-centradas, que el historiador cree detectar en la raíz de la negativa de los autores a 'reconocer la posibilidad de error propio' (Boring, 1929/1963b).

Tal concepción de las controversias condice con el principio metacientífico normativo rector común a la mayoría de los primeros psicólogos-historiadores acerca del carácter deseable de la ciencia. Este principio fue expresado tempranamente por Boring: "Si la ciencia [cursivas añadidas] es la búsqueda desapasionada de la verdad a través del método empírico, ¿puede florecer a pesar de la pasión?" (Boring, 1929/1963b, p. 78). La respuesta del autor es ilustrativa de la cuestión que aquí se aborda: dado que la controversia involucra emoción y pasión que nublan el juicio de los científicos y los fija a sus propias opciones, "la discusión científica debería ser [cursivas añadidas] desapasionada, no sólo en forma sino en contenido, puesto que de otra forma 
el progreso hacia la verdad es entorpecido" (Boring, 1929/1963b, p. 78). Según tal aserto, la verdad sería más fácilmente alcanzable si las 'grandes mentes' no tuvieran que trabarse en discusiones y debates.

\section{La Controversia según la Historiografía Crítica: la Discusión y el Debate como Constitutivas e Invariantes de la Ciencia}

Las controversias históricas de la psicología fueron conceptualizadas de una forma radicalmente disímil por las obras de los historiadores posteriores a Boring. $\mathrm{Si}$ consideramos que los historiadores posteriores a 1970 comenzaron a incorporar de forma sistemática teorías, metodologías y recursos de análisis de la nueva filosofía y sociología del conocimiento y de la ciencia, es comprensible que la definición, el estudio y la valoración de las controversias psicológicas cambiasen concomitantemente con la redefinición de cuestiones historiográficas, metacientíficas y epistemológicas basales, como las referidas a la naturaleza de la ciencia psicológica, al carácter ontológico de los objetos psicológicos y a la relación entre las prácticas de los científicos y los referentes de sus teorías (Fierro, 2015b; Louw, 2003; Polanco \& Fierro, 2015; Talak, 1997). Reflexivamente, esta variación afectó a la naturaleza de las propias reconstrucciones históricas, como objeto de estudio y producción de los propios historiadores.

\section{La Naturaleza Problemática y Controversial de la Reconstrucción de la Historia de la Psicología}

La nueva historia de la psicología tematizó y revisó la historiografía clásica de la psicología. Por un lado, los perceptos teóricos y metodológicos de dicha historia fueron equilibrados (cuando no reemplazados) por orientaciones colectivistas (o grupalistas), historicistas, no-celebratorias (críticas) realizadas tanto por psicólogos formados sistemáticamente en historia de la ciencia, como por historiadores profesionales (Capshew, 2014; Danziger, 1994; Furumoto, 1989; Woodward, 1980).

La perspectiva individualista en historia y sociología de la ciencia defendida por Boring fue criticada por perspectivas teóricas de índole colectivista o grupalista, y por perspectivas metodológicas que priorizaron el relevamiento empírico de las fuentes históricas primarias, circunscribiendo y limitando de esta forma las hipótesis a priori respecto de la motivación imputada a los psicólogos y a las escuelas psicológicas históricas (Danziger, 1979; Wettersten, 1975).

Adicionalmente, se analizaron críticamente las raíces teóricas y profesionales de los historiadores clásicos. Respecto de la historiografía de Boring se ha argumentado que buscó legitimar los principios de su grupo profesional de referencia y pertenencia-el de los psicólogos básicos experimentales y operacionistas(Ash, 1983; Danziger, 1990; Gruba-McCallister, 1978).

Si bien múltiples autores han defendido un modelo no-dicotómico de análisis histórico (Buss, 1975; Danziger, 1984; Shapin, 1982; Vilanova, 1995), gran parte de la historiografía crítica es claramente 'externalista' o 'contextualista', puesto que sus indagaciones históricas suelen remitirse a los ámbitos intelectuales y socio-culturales más amplios para explicar la historia teórica y profesional de la disciplina (Mendelsohn, 1977).

Así, como efecto de los cambios historiográficos hacia 1970, se reconoce progresivamente la naturaleza controversial de la historia de la psicología, fruto del carácter problemático (esto es, conjetural y discutible) y reconstructivo (esto es, a posteriori de los hechos y, consecuentemente, dependiente de intereses y presupuestos) de toda narrativa histórico-científica (Weimer, 1974). Tales ideas (y a nivel más general, aquellos cambios) constituyen un claro caso de apropiación de ciertos asertos de la sociología de la ciencia y de los estudios sociales de la ciencia por parte de la historia e historiografía de la psicología (Buchdahl, 1965; Fierro, 2015b; Shapin, 1982; Ziman, 1985).

\section{Perspectivas Sociológicas y Psico-Sociológicas sobre Controversias Históricas}

Las nuevas tendencias históricas redefinieron el objeto de estudio de los psicólogos-historiadores. Por un lado, incidencias sociológicas han llevado a concebir a la disciplina como un conjunto heterogéneo de grupos humanos regulados socialmente, enfatizándose la dimensión intersubjetiva de la empresa científica. Esto lleva a definir a la historia de la disciplina como una sociología del pensamiento cuyos 'sujetos históricos' son colectivos teóricos -las escuelas teóricas y metodológicas- (Harvey, 1965; Watson, 1975) y sociales -los grupos de psicólogos profesionales con sus instituciones, experticia, legitimación e intereses intelectuales (Buss, 1975; Danziger, 1984; Sokal, 1984). Aquí se incorporan de la filosofía de la ciencia de segunda mitad del siglo XX el análisis de la dimensión 
social de los grupos científicos (Campbell, 1979/1988; Shapin, 1982) y la pragmática de la investigación y comunicación científica (Ash, 1987).

La nueva historiografía enfatiza la faz pragmática de la ciencia: la exposición pública de argumentos, la discusión y regulación social, la vinculación científica y profesional y, por tanto, la retórica y la comunicación (Ash, 1993; Danziger, 1979, 1990; Leary, 1987; van Strien, 1993). Del énfasis en la negociación y en la actividad real y concreta de los psicólogos se extrae que el conocimiento psicológico no es estrictamente el producto de la aplicación de metodologías concretas a datos empíricos, sino, además, el conjunto de pretensiones de conocimiento que para verificarse y legitimarse requieren ser sometidas al escrutinio y análisis no sólo de la realidad sino también de la comunidad científica como circuito de pares expertos (Danziger, 1995; van Strien, 1993). De aquí la importancia de las discusiones y los debates públicos (la controversia científica) para analizar la naturaleza, gesta y desarrollo del conocimiento psicológico.

La nueva historia de la psicología ha concebido a la controversia de forma progresiva y paulatina como estructural al desarrollo histórico de la psicología. Esto implica una revalorización de tales debates (lo que los hace objeto específico de interés e indagación específicas) y, por otro, una redefinición de tales instancias, ya no como choque irracional de científicos ego-centrados y pasionales sino como sucesos inherentes a la dinámica científica que involucran, entre otras cosas, intereses intelectuales específicos y contrapuestos, y criterios epistemológicos y metodológicos disímiles.

Respecto de la revalorización, se ha sostenido que "la controversia no sólo es útil, sino probablemente ineludible, puesto que surge de las propiedades esenciales de nuestro funcionamiento cognitivo" (Henle, 1973, p. 47). En este sentido, y siguiendo a la sociología histórica de la ciencia (Böhme, 1977; Schaffer, 1985), se argumenta que las controversias son acontecimientos históricos vitales en tanto que en ellas se explicitan las reglas, normas, valores y prescripciones científicas normalmente implícitas. De aquí que se sostenga que las controversias no deberían removidas de las reconstrucciones históricas, sino incluidas como instancias legítimas y ejemplares del desarrollo de la ciencia que evidencian el carácter problemático de ciertos núcleos temáticos y principios teórico-metodológicos de la psicología.

Respecto de la redefinición, según los historiadores contemporáneos las controversias no son ejemplos de la pasión, irracionalidad o perseveración mental de los psicólogos: son instancias empíricas y parcialmente racionales donde se discuten explícitamente resultados e, implícitamente, modelos científicos y criterios metodológicos y epistemológicos acerca de la psicología. Los historiadores sostienen que la perseveración, tenacidad y disposición a prolongar el debate son invariantes y estructurales de la dimensión social de la ciencia y, desde una perspectiva epistemológica naturalista, no necesariamente perniciosas. Sólo descontextualizadas las controversias aparecen como el intercambio fútil de científicos necios: si se contextualizan los argumentos en debate en el sistema teórico, metodológico y social de cada autor, la naturaleza de la controversia revela su carácter racional, y es entonces factible de elucidarse. Sólo a partir del whiggismo de la historiografía clásica puede argumentarse que la controversia entre Wundt y Stumpf fuera acerca de una mera diferencia sobre tonalidades, o que el debate entre Baldwin y Titchener sobre reacciones sensorio-motrices tuviera origen en el ego de los autores. Sólo si la controversia se juzga a partir del conocimiento actual y a partir de una teoría de la motivación pre-establecida (como por ejemplo la freudiana) puede sostenerse de Wundt y Stumpf que "ambos no podían tener razón; ambos lo sabían [...] Simplemente existe una tendencia perseverante en el pensamiento científico" (Boring, 1929/1963b, p. 76), o que el enfrentamiento podía ser reducido a la cerrazón mental producto de la necesidad de los autores de realizar "promoción y propaganda" (Boring, 1929/1963b, p. 79).

Propuestas historiográficas críticas han propuesto que el debate Baldwin-Titchener involucró cuestiones que se derivaban de la discusión acerca del carácter científico de la psicología y de la metodología más adecuada para garantizar tal carácter (Danziger \& Shermer, 1994). Se ha remarcado que a ambos divergían en cuanto a sus intereses investigativos, sus objetos de estudio y sus escuelas teóricas de pertenencia (Böhme, 1977). En este sentido, según analizan Krantz (1969), Henle (1973) y Danziger y Shermer (1994) desde una perspectiva psicosociológica, el debate, lejos de ser el encuentro de dos 'egotistas', constituye el choque de dos escuelas (estructuralismo y funcionalismo) emergentes, disímiles y contrapuestas. La controversia es función tanto del contexto que conforman los principios y directrices de tales escuelas, como del efecto modulador que ejerce dicho contexto sobre los hallazgos empíricos de los científicos ${ }^{6}$. Así, las escuelas

\footnotetext{
6 No hay referencias o tematizaciones explícitas sobre dichas controversias en las obras de los principales historiadores de primera mitad del siglo XX. Heidbreder (1933/1960, pp. 190) menciona
} 
influenciarían tanto a través de las normas implícitas y a través de la conformidad a que mueven (Harvey, 1965; Watson, 1975) como a través de las normas explícitas que constituyen el cuerpo normativo de los movimientos psicológicos: "Que diferentes hallazgos se obtengan en diferentes laboratorios es resultado de las condiciones establecidas, de las tareas presentadas, de la instrucción de los sujetos y de los experimentadores, entre otras" (Henle, 1973, p. 51). Algo semejante puede decirse acerca de las controversias entre la psicofisiología wundtiana y la psicología alemana de Ash, Bühler y Külpe (Kusch, 1995), y acerca de las controversias sobre del estatus institucional de la primera psicología científica en las que Wundt participó activamente (Araujo, 2013; Ash, 1980). La historia social de la psicología ha vinculado significativamente los contextos sociales e institucionales de tales controversias, las diversas estructuras sociales de los institutos de investigación y de las instancias investigativas, y las propuestas programáticas elaboradas por autores germanos para la psicología científica.

Los debates y enfrentamientos científicos públicos llevarían efectivamente al desarrollo y al cambio teórico, y las controversias no se reducirían a discusiones semánticas sobre superficialidades metodológicas. De aquí que la propia proliferación de escuelas, concebida por la historiografía clásica (Boring, 1929/1963b, 1955) como la negación irracional de la sabiduría generacional, se explique alternativamente por la variedad de normas y valores estructurantes del trabajo científico, de prácticas profesionales basales en la legitimación e institucionalización de la disciplina y de problemáticas (Danziger, 1984) a que las escuelas han pretendido ser respuestas tentativas (independientemente que ciertas escuelas hayan olvidado progresivamente el carácter conjetural de su conocimiento y la naturaleza parcial de sus conocimientos). sólo en limitadas ocasiones a J. M. Baldwin, y respecto de su controversia con Titchener, declara que tal debate fue acerca de la 'interpretación', agregando sucintamente que dicha controversia evidenció dos formas de comprender la psicología. Woodworth (1938/1961) no menciona a Baldwin en ninguna sección de su manual. Tampoco parece hacerlo Murphy (1929/1964). Este último, sin embargo, sí releva ciertos puntos de desencuentro entre Wundt, Titchener y Würzburg (Murphy, 1929/1964, pp. 223-230) pero nuevamente de forma expositiva, descriptiva y paralelista (esto es, sin puntualizar los debates entre autores).

\section{Conclusiones}

La historiografía clásica, abrevando en principios explicativos de las profesiones de los historiadores, a menudo produjo una historia de la psicología legitimante, presentista y justificacionista. Dados los implícitos de tal historiografía, la posibilidad de inexistencia (aún temporaria) de un progreso lineal, acumulativo e inexorable en la historia constituyó una anomalía problemática que incluir en las narrativas históricas. En psicología, tal posibilidad se encarnó de forma concreta en las controversias científicas.

La 'nueva historia' (o historia crítica), cuyo programa historiográfico pretendía equilibrar la historiografía antecesora, revalorizó y reformuló las instancias controversiales propias de la historia de la disciplina. Según la historia clásica y su visión normativa positivista de la ciencia las controversias eran reducibles a la intromisión indeseable de cierta irracionalidad pasional y emotiva que cegaba a aquellos científicos que no vigilaban sus propios sesgos y tendencias inconcientes. $\mathrm{La}$ historiografía crítica de la psicología reformuló tales planteos, incorporando recursos específicos de la filosofía e historiografía de la ciencia post-kuhniana-como el giro pragmático y la historia social de la ciencia- y de la sociología del conocimiento y de la ciencia -en especial el énfasis en la naturaleza y raigambre contextual y social del conocimiento científico-. Así, las controversias serían instancias estructurales en la dinámica científica, debido al carácter estructural de ciertos sesgos cognitivos en los científicos, y a la naturaleza intrínseca de ciertas normas y valores al interior de las escuelas psicológicas. Así, las controversias se presentan como fértiles casos de estudio para reconstruir de forma plausible y veraz el pasado disciplinar-objetivo de la historia de la ciencia y de historia la psicología-, y para reflexionar acerca de las complejas relaciones entre la práctica de los científicos, sus objetos de estudio, sus pretensiones de conocimiento y el contexto intelectual y societal más general.

Según esta perspectiva, la actividad de los psicólogos se revela constreñida por prescripciones que sólo se explicitan durante las controversias científicas públicas, en las argumentaciones y contraargumentaciones que son su vehículo conceptual. En este sentido, las controversias se prestan a la historiografía como instancias clave para analizar los principios -teóricos, metodológicos y metacientíficos-que regulan de forma implícita la actividad científica.

Tal como la historia no es un ornamento o accesorio para la psicología contemporánea -en tanto 
constituye un campo meta-reflexivo de crítica y de evaluación constante-, las controversias históricas no constituirían errores, equivocaciones o perseveraciones del pensamiento de psicólogos que "no sabían que estaban equivocados'. Tales controversias históricas constituyen ejemplares notables, y dignos de estudios en profundidad, susceptibles de evidenciar la naturaleza tanto de la disciplina como de las relaciones intersubjetivas de sus agentes sociales. Esta revalorización de las instancias públicas de debate, muy propia de los estudios sociales de la ciencia -y específicamente del Programa Empírico del Relativismo de la sociología de la ciencia- ha representado una incursión estimulante en la historiografía contemporánea de la psicología. Es factible que la sistematización de los estudios sobre tales controversias en psicología, en conjunto con la sistematización del anudamiento entre la historia de la psicología y los estudios sociales de la ciencia y la tecnología, redunde en más y mayores beneficios para ambos campos, con el objetivo de clarificar variadas cuestiones meta-teóricas y meta-científicas de gran interés y relevancia para todo psicólogo contemporáneo, científico a la vez que profesional.

\section{Referencias}

Araujo, S. (2013). O Manifesto dos filósofos alemães contra a psicologia experimental: introdução, tradução e comentários. Estudos e Pesquisas em Psicologia, 13(1), 298-311. Recuperado de http:// www.revispsi.uerj.br/v13n1/artigos/pdf/v13n1a18.pdf

Ash, M. (1980). Wilhelm Wundt and Oswald Külpe on the Institutional Status of Psychology: An Academic Controversy in Historical Context. En W. Bringmann \& R. Tweney (Eds.), Wundt Studies (pp. 396-421). Toronto: Hogrefe.

Ash, M. (1983). The Self-Presentation of a Discipline: History of Psychology in the United States between Pedagogy and Scholarship. En L. Graham, W. Lepenies \& P. Weingart (Eds.), Functions and Uses of Disciplinary Histories (pp. 143-189). Dordretch: Springer. doi: 10.1007/978-94-009-7035-9_7

Ash, M. (1987). Introduction. En M. Ash \& W. Woodward (Eds.), Psychology in Twentieth-Century Thought and Society (pp. 1-12). Nueva York: Cambridge University Press.

Ash, M. (1992). Historicizing Mind Science: Discourse, Practice, Subjectivity. Science in Context, 5(2), 193-207. doi:10.1017/ S0269889700001150

Ash, M. (1993). Rhetoric, Society, and the Historiography of psychology. En H. Rappard, P. Van Strien, L. Mos \& W. Baker (Eds.), Annals of Theoretical Psychology, Vol. 8 (pp. 49-57). Nueva York: Plenum Press. doi:10.1007/978-1-4615-2982-8_3

Blight, J. (1981). Toward the Reconstruction of Psychology and Its Historiography. Journal of the History of the Behavioral Sciences, 17(1), 136-143. doi:10.1002/1520-6696(198101)17:1<136::AIDJHBS2300170116>3.0.CO;2-N

Böhme, G. (1977). Cognitive Norms, Knowledge-Interests and the Constitution of the Scientific Object: A Case Study in the Functioning of Rules of Experimentation. En E. Mendelsohn, P.
Weingart \& R. Whitley (Eds.), The Social Production of Scientific Knowledge (pp. 129-142). Dordrecht: Reidel. doi:10.1007/97894-010-1186-0_6

Boring, E. (1929/1963a). Interpretation. En R. Watson \& D. Campbell (Eds.), History, Psychology, and Science: Selected Papers by Edwin Boring (pp. 26-28). John Wiley: Nueva York.

Boring, E. (1929/1963b). Psychology of Controversy. En R. Watson \& D. Campbell (Eds.), History, Psychology, and Science: Selected Papers by Edwin Boring (pp. 67-84). John Wiley: Nueva York.

Boring, E. (1954). Psychological Factors in the Scientific Progress. American Scientist, 42(4), 639-645. doi:10.2307/27826574

Boring, E. (1955). Dual Role of the Zeitgeist in the Scientific Creativity. Scientific Monthly, 80(2), 101-106. doi:10.2307/21367

Brush, S. (1974). Should the History of Science Be Rated X? Science, 183(4130), 1164-1172. Recuperado de http://oregonstate.edu/ instruct/hsts414/doel/SB_H_S_rated_X.pdf

Bucchi, M. (2004). Science in Society. An Introduction to Social Studies of Science. London: Routledge.

Buchdahl, G. (1965). A Revolution in Historiography of Science. History of Science, 4, 55-69. doi:10.1177/007327536500400103

Buss, A. (1975). The Emerging Field of the Sociology of Psychological Knowledge. American Psychologist, 30(10), 988-1002. doi:10.1037/0003-066X.30.10.988

Buss, A. (1978). The structure of psychological revolutions. Journal of the History of the Behavioral Sciences, 14(1), 57-64. doi:10.1002/ 1520-6696(197801)14:1<57::AID-JHBS2300140109>3.0.CO;2-7

Campbell, D. (1979/1988). A Tribal Model of the Social System Vehicle Carrying Scientific Knowledge. En D. Campbell (Comp.), Methodology and Epistemology for Social Sciences: Selected Papers (pp. 489-503). Chicago: Chicago University Press.

Capshew, J. (2014). History of Psychology since 1945: A North American Review. En R. Backhouse \& P. Fontaine (Eds.), A Historiography of the Modern Social Sciences (pp. 144-182). Nueva York: Cambridge University Press. doi:10.1017/CBO9781139794817

Collins, H. (1981). Stages in the Empirical Programme of Relativism. Social Studies of Science, 11, 3-10. doi:10.1177/ 030631278101100101

Collins, H. (1983). An Empirical Relativist Programme in the Sociology of Scientific Knowledge. En K. Knorr-Cetina \& M. Mulkay (Eds.), Science Observed (pp. 85-113). London: Sage.

Collins, H. \& Pinch, T. (1993). The Golem: What everyone should know about science. Cambridge: Cambridge University Press.

Danziger, K. (1979). The Social Origins of Modern Psychology. En A. Buss (Ed.), Psychology in Social Context (pp. 27-45). Nueva York: Irvington.

Danziger, K. (1984). Towards a Conceptual Framework for a Critical History of Psychology. Revista de Historia de la Psicología, 5(1), 99-107. Recuperado de http://www.revistahistoriapsicologia.es/ revista/1984-vol-5-n\%C3\%BAm-1-2/

Danziger, K. (1990). The Social Context of Research Practice and the History of Psychology. En W. Baker, R. van Hezewijk, M. Hyland \& S. Terwee (Eds.), Recent Trends in Theoretical Psychology, Vol. II (pp. 297-303). Nueva York: Springer-Verlag. doi:10.1007/978$1-4613-9688-8$

Danziger, K. (1994). Does the history of psychology have a future?. Theory and Psychology, 8(5), 467-484. doi: $10.1177 / 0959354394044001$

Danziger, K. (1995). The Production of Psychological Knowledge by Experts. Cuadernos Argentinos de Historia de la Psicología, 1(1-2), 259-273.

Danziger, K. \& Shermer, P. (1994). The varieties of replication: A historical introduction. En J. Valsiner, R. van der Veer \& M. van Ijzendoorn (Eds.), Reconstructing the mind: Replicability in research on human development (pp. 17-36). Norwood: Ablex. 
Diéguez, A. (2004). Los Estudios sobre Ciencia, Tecnología y Sociedad. Una Panorámica General. En J. Atencia \& A. Diéguez (Eds.), Tecnociencia y Cultura a Comienzos del Siglo XXI (pp. 53-86). Málaga: Universidad de Málaga.

Fernández, A. (2009). El Constructivismo Social en la Ciencia y la Tecnología: Las consecuencias no previstas de la ambivalencia epistemológica. ARBOR Ciencia, Pensamiento y Cultura, 185(738), 689-703. doi:10.3989/arbor.2009.738n1046

Fierro, C. (2015a). La Historia de la Psicología y su Enseñanza a 40 años de 'Should the History of Science Be Rated X?' de Stephen Brush. Estudos e Pesquisas em Psicologia, 15(1), 310-330. Recuperado de http://www.e-publicacoes.uerj.br/index.php/revispsi/ article/view/16076/12095

Fierro, C. (2015b). La Historiografía de la Psicología: Historia Clásica, Historia Crítica y la Recepción de los Estudios Sociales de la Ciencia. Revista de Historia de la Psicología, 36(2), 67-94. Recuperado de http://www.revistahistoriapsicologia.es/ revista/2015-vol-36-n\%C3\%BAm-2/

Fierro, C. (En prensa). Un Encuentro Problemático: Historiografía de la Psicología y Enseñanza de la Historia de la Psicología en Estados Unidos. En T. Caycho Rodríguez (Ed.), Estudios sobre historia de la psicología en el Perú y Latinoamérica. Perú: Universidad Inca Garcilaso de la Vega.

Fierro, C. \& Visca, J. (En prensa). Revisión de un concepto central en la Formación y Enseñanza en Historia de la Psicología: Las escuelas psicológicas desde la Historia, la Sistematología y la Sociología de la Psicología. En AA.VV., Avances y Desafíos para la Psicología. San Luis, Argentina: Universidad Nacional de San Luis.

Friedman, R. (1967). Edwin G. Boring's "mature" view of the science of science in relation to a deterministic personal and intellectual motif. Journal of the History of the Behavioral Sciences, 3(1), 17-26. Recuperado de http://onlinelibrary.wiley. com/doi/10.1002/1520-6696\%28196701\%293:1\%3C17::AIDJHBS2300030103\%3E3.0.CO;2-\%23/abstract

Furumoto, L. (1989). The new history of psychology. En I. Cohen (Ed.), The G. Stanley Hall Lecture Series, Vol. 9 (pp. 5-34). Washington, D.C: APA.

Gallegos, M. (2014). Thomas Kuhn y su vinculación con la psicología: Un homenaje de despedida. Revista de Historia de la Psicología, 35(2), 65-92. Recuperado de http://www.revistahistoriapsicologia.es/revista/2014-vol-35-n\%C3\%BAm-2

Golinski, J. (1990). The Theory of Practice and the Practice of Theory: Sociological Approaches in the History of Science. Isis, 81, 492-505. doi:10.1086/355457

Gruba-McCallister, F. (1978). Efficient Causality in Boring's Work and Thought: A Case of One-Sided Determinism. Journal of the History of the Behavioral Sciences, 14(3), 207-212. doi:10.1002/1520-6696(197807)14:3<207::AIDJHBS2300140303>3.0.CO;2-Q

Harris, B. (1980). Ceremonial Versus Critical History of Psychology. American Psychologist, 35(2), 218-219. doi:10.1037/0003066X.35.2.218

Harvey, O. (1965). The History of Psychology as Sociology of Thought. Journal of the History of the Behavioral Sciences, 1(2), 196-202. doi:10.1002/1520-6696(196504)1:2<196::AIDJHBS2300010213>3.0.CO;2-N

Heidbreder, E. (1933/1960). Psicologías del siglo XX. Buenos Aires: Paidós.

Henle, M. (1973). On Controversy and Its Resolution. En M. Henle, J. Jaynes \& J. Sullivan (Eds.), Historical Conceptions of Psychology (pp. 47-59). Nueva York: Springer.

Jaraba-Barrios, B. \& Mora-Gámez, F. (2010). Reconstruyendo el objeto de la crítica: sobre las posibles confluencias entre psicología crítica y estudios sociales de la ciencia y la tecnología. Revista
Colombiana de Psicología, 19(2), 225-239. Recuperado de http:// www.redalyc.org/articulo.oa?id=80415435006

Klappenbach, H. (2000). Historia de la historiografía de la psicología. En J. Ríos, R. Ruiz, J. Stagnaro \& P. Weissman (Eds.), Psiquiatría, Psicología y Psicoanálisis: Historia y Memoria (pp. 238-268). Buenos Aires: Polemos.

Klappenbach, H. (2013). Aportes de la Historia de la Psicología a la Integración y Diversidad en Psicología. En J. Alchieri \& J. Barreiros (Org.), Confêrencias do XXXIV Congreso Interamericano de Psicología (pp. 83-98). Brasilia: SBPOT.

Krantz, D. (1969). The Baldwin-Titchener controversy. En D. Krantz (Ed.), Schools of Psychology (pp. 1-19). Nueva York: Appleton Century Croft.

Kuhn, T. (1962/1970). The Structure of Scientific Revolutions. Chicago: The University of Chicago Press.

Kusch, M. (1995). Recluse, Interlocutor, Interrogator: Natural and Social Order in Turn-of-the-Century Psychological Research Schools. Isis, 86(3), 419-439. Recuperado de http://www.jstor. org/stable/235021?seq=1\#page_scan_tab_contents

Leary, D. (1987). Telling likely stories: The rhetoric of the New Psychology, 1880-1920. Journal of the History of the Behavioral Sciences, 23(4), 315-331. doi:10.1002/15206696(198710)23:4<315::AID-JHBS2300230402>3.0.CO;2-V

Louw, J. (2003). In Search of Method. En A. Brock, J. Louw \& W. Van Hoorn (Eds.), Rediscovering the history of psychology: Essays inspired by the work of Kurt Danziger (pp. 33-52). Nueva York: Springer. doi: $10.1007 / \mathrm{b} 106634$

Mendelsohn, E. (1977). The Social Construction of Scientific Knowledge. En E. Mendelsohn, P. Weingart \& R. Whitley (Eds.), The Social Production of Scientific Knowledge (pp. 3-26). Dordrecht: Reidel.

Murphy, G. (1929/1964). Introducción histórica a la Psicología contemporánea. Buenos Aires: Paidós.

Polanco, F. \& Fierro, C. (2015). Recepción de la Sociología del Conocimiento en la Historia de la Psicología. Revista de Psicología de Arequipa, 5(1), 13-35. Recuperado de http://www.academia. edu/11944275/Recepci\%C3\%B3n_de_la_sociolog\%C3\%ADa del_conocimiento_y_de_la_ciencia_en_la_historia_de_la psicolog\%C3\%ADa_Reception_of_the_Sociology_of_Knowledge_and_the_Sociology_of_Science_in_the_History_of_Psychology

Richards, G. (1987). Of What is History of Psychology a History? The British Journal for the History of Science, 20(2), 201-211. doi: $10.1017 /$ S0007087400023748

Ross, D. (1969). The "Zeitgeist" and American Psychology. Journal of the History of the Behavioral Sciences, 5(3), 256-262. doi:10.1002/1520-6696(196907)5:3<256::AIDJHBS2300050305>3.0.CO;2-5

Schaffer, S. (1985). What is the History of Science?. History Today, $35,49-50$

Shapin, S. (1982). History of Science and Its Sociological Reconstructions. History of Science, 20(3), 157-211. doi:10.1007/97894-009-4498-5_18

Sokal, M. (1984). James McKeen Cattell and American Psychology in the 1920s. En J. Brozek (Ed.), Explorations in the History of Psychology in the United States (pp. 273-323). Nueva Jersey: Associated University Presses.

Talak, A. (1997). Comentarios sobre la historia crítica de la psicología y la sociología del conocimiento. Anuario de Investigaciones de la Universidad de Buenos Aires, 5, 572-581. Recuperado de http:// www.psi.uba.ar/docentes_graduados.php?var=investigaciones/ revistas/anuario/anteriores/anuario5/historia.php

van Strien, P. (1993). The Historical Practice of Theory Construction. En H. V. Rappard, P. van Strien, L. Mos \& W. Baker (Eds.), 
Annals of Theoretical Psychology, Vol. 8 (pp. 149-227). Nueva York: Plenum Press.

Vilanova, A. (1990). La raíz social del conocimiento psicológico. Mar del Plata: Universidad Nacional de Mar del Plata.

Vilanova, A. (1995). El dilema olvidado de la psicología latinoamericana. Cuadernos Argentinos de Historia de la Psicología, 1(1/2), 83-99.

Vilanova, A. (1997a). La historia de la psicología y su sentido curricular. Clepios, 3(2), 18-23.

Vilanova, A. (1997b). Las deudas de la Psicología del Cono Sur. Acta Psiquiátrica y Psicológica de América Latina, 43(2), 103-111.

Watson, R. (1975). Prescriptive Theory and the Social Sciences. En K. Knorr, H. Strasser \& H. G. Zilian (Eds.), Determinants and Controls of Scientific Development (pp. 11-36). Dordretch: Reidel.

Weimer, W. (1974). The History of Psychology and Its Retrieval from Historiography: I. The Problematic Nature of History. Science Studies, 4(3), 235-258. Recuperado de www.researchgate.net/
publication/261782618_The_History_of_Psychology_and_Its Retrieval_from_Historiography_I._The_Problematic_Nature_of_History

Weimer, W. (1979). Notes on the Methodology of Scientific Research. Nueva York: Erlbaum.

Wettersten, J. (1975). The historiography of scientific psychology: A critical study. Journal of the History of the Behavioral Sciences, 11(2), 157-171. doi:10.1002/1520-6696(197504)11:2<157::AIDJHBS2300110207>3.0.CO;2-5

Woodward, W. (1980). Toward a Critical Historiography of Psychology. En J. Brozek \& L. Pongratz (Eds.), Historiography of Modern Psychology (pp. 29-70). Toronto: Hogrefe.

Woodworth, R. (1938/1961). Contemporary Schools of Psychology. Calcuta: Asia Publishing House.

Young, R. (1966). Scholarship and the History of the Behavioural Sciences. History of Science, 5, 1-51. doi:10.1177/007327536600500101

Ziman, J. (1985). What is the History of Science? History Today, $35,52-53$. 